\title{
Marked Baseline Fetal Heart Rate Variability
}

National Cancer Institute

\section{Source}

National Cancer Institute. Marked Baseline Fetal Heart Rate Variability. NCI Thesaurus.

Code C111765.

A baseline fetal heart rate with an amplitude range that is greater than 25 beats per minute. 